\title{
RESOLUÇÃO INTEGRADA DE PROBLEMAS DO PLANEJAMENTO DO TRANSPORTE PÚBLICO: FOCO NA TABELA DE HORÁRIOS E NO ESCALONAMENTO DE VEÍCULOS COM FROTA HETEROGÊNEA*
}

\author{
INTEGRATED RESOLUTION OF PUBLIC TRANSPORT PLANNING \\ PROBLEMS: FOCUS ON TIMETABLING AND VEHICLE \\ SCHEDULING WITH HETEROGENEOUS FLEET
}

\author{
Data de submissão: $23 / 11 / 2014$ \\ Aceite: 16/06/2016 \\ Monize Sâmara Visentini ${ }^{1}$ \\ Olinto César Bassi de Araújo² \\ Denis Borenstein ${ }^{3}$ \\ Alberto Francisco Kummer Neto ${ }^{4}$
}

\section{RESUMO}

Neste artigo, propõe-se um novo modelo de Programação Linear Inteira, baseado em uma rede tempo-espaço, que integra os problemas de geração da tabela de horários e o escalonamento de veículos com frota heterogênea. Um diferencial dessa abordagem consiste na consideração da demanda para a redefinição da tabela de horários e para o escalonamento dos veículos, fator raramente aplicado nos modelos de otimização do sistema de transporte. Foram utilizadas instâncias reais e aleatórias de grande porte. Os resultados indicam que o modelo pode contribuir para a otimização do planejamento do transporte público, tendo em vista que possibilita economias significativas no número de veículos escalonados. Além disso, como os intervalos de alteração da tabela de horários são bastante curtos, obtêm-se alterações sutis, modificando minimamente a rotina dos passageiros, o que possibilita a aplicação dessa abordagem ao contexto real.

Palavras-chave: Escalonamento; Ônibus; Otimização.

\footnotetext{
* Os autores agradecem ao apoio financeiro do CNPq e do Estado do Rio Grande do Sul, por intermédio da FAPERGS, Processo №.: 16/2551-0000235-5. 1 Possui Graduação em Administração pela Universidade Federal de Santa Maria, UFSM, mestrado em Mestrado em Administração pela Universidade Federal de Santa Maria, UFSM, e doutorado em Administração pela Universidade Federal do Rio Grande do Sul, UFRGS. Cerro Largo, Brasil E-mail: monize.visentini@uffs.edu.br

2 Possui graduação em Licenciatura Plena em Matemática pela Universidade da Região da Campanha, URCAMP, mestrado em Modelagem Matemática pela Universidade Regional do Noroeste do Estado do Rio Grande do Sul, UNIJUI e doutorado em Engenharia Elétrica pela Universidade Estadual de Campinas, UNICAMP. Atualmente é Professor de Ensino Básico, Técnico e Tecnológico do Colégio Técnico Industrial de Santa Maria - Universidade Federal de Santa Maria. Santa Maria, Brasil. E-mail: olinto@ctism.ufsm.br

3 Possui graduação em Engenharia Naval pela Universidade Federal do Rio de Janeiro, UFRJ, mestrado em Administração pela Universidade Federal do Rio Grande do Sul, UFRGS e doutorado em Pesquisa Operacional pela University of Strathclyde, STRATH. Atualmente é professor associado da Universidade Federal do Rio Grande do Sul. Porto Alegre, RS - Brasil. E-mail: denisb@ea.ufrgs.br 4 Possui Graduação em Ciência da Computação pela Universidade Federal de Santa Maria, UFSM, mestrado em Informática pela Universidade Federal de Santa Maria, UFSM, doutorado em andamento em PPGC - Programa de Pós Graduação em Computação UFRGS pela Universidade Federal do Rio Grande do Sul, UFRGS, Porto Alegre. E-mail: alberto@inf.ufsm.br
} 


\begin{abstract}
In this paper, we propose a new Integer Linear Programming model, based on a time-space network that integrates the timetable generation problem and the vehicle scheduling problem with heterogeneous fleet. A difference of this approach consists in considering the demand for the timetable redefinition and the vehicle scheduling, factor rarely applied in optimization models of the transportation system. We applied real and large random instances. The results indicate that the model may contribute to optimizing the public transport planning leading to significant savings in terms of scheduled vehicles. Moreover, as the timetable changes are fairly short, it is slightly modified, minimally modifying the passengers routine, which enables the application of these approaches to real context.
\end{abstract}

Keywords: Scheduling; Bus; Optimization.

\title{
1 INTRODUÇÃO
}

O problema de escalonamento de veículos (Vehicle Scheduling Problem - VSP), amplamente estudado nas últimas décadas, possui escassez de estudos, considerando frota heterogênea na literatura de transportes, em comparação à frota homogênea (Ceder, 2011). No contexto do transporte público, uma frota heterogênea compreende diferentes tipos de ônibus que variam em capacidade, velocidade ou equipamentos. Problemas de escalonamento de veículos com frota heterogênea (Vehicle Type Scheduling Problem - VTSP) são classificados por Ceder (2011) como aqueles que integram a programação de veículos e a melhor escolha do tipo de veículo necessário para cada viagem, mantendo o nível da qualidade do serviço prestado. No transporte público, muitos fatores influenciam a inclusão da frota heterogênea, como a substituição da frota, novas tecnologias para os ônibus, oportunidades de mercado, demanda crescente, reutilização em novas linhas de ônibus de veículos desativados etc. Percebe-se, assim, a importância de se considerar esse aspecto quando do escalonamento dos veículos no planejamento do transporte público.

A programação da frota heterogênea não está somente relacionada ao interesse econômico das empresas de ônibus, influindo também na qualidade da viagem oferecida aos passageiros e nas características do trânsito urbano. Uma viagem feita por um ônibus articulado, por exemplo, pode atender à demanda de duas viagens consecutivas realizadas por ônibus menores, sem prejuízos aos passageiros. Ao tratar a frota heterogênea como um problema, é interessante que se analise a demanda que deverá ser atendida, tendo em vista que se podem direcionar os tipos de veículos conforme o número de passageiros previstos para cada viagem. Outra consequência do uso da frota heterogênea é a possibilidade de se alterar, sutilmente, a tabela de horários e melhorar ainda mais o escalonamento dos veículos utilizados, integrando as duas primeiras etapas do planejamento do transporte público, definidas por Freling, Wagelmans e Paixão (1999) como: a geração da tabela de horários, o escalonamento de veículos, o escalonamento da tripulação e a rotação da tripulação. Otimizando simultaneamente os problemas de geração da tabela de horários e de escalonamento de veículos com frota heterogênea, obtém-se mais flexibilidade nos horários de partida das viagens e maior compatibilidade de viagens, possibilitando reduções no custo total do planejamento.

Baseando-se nessas considerações, neste artigo propõe-se um novo modelo de Programação Linear Inteira (PLI), baseado em uma rede tempo-espaço (Time-Space Network, TSN) que integra os problemas de geração da tabela de horários e o VTSP. Um diferencial dessa abordagem consiste na consideração da demanda para a redefinição da tabela de horários e para o escalonamento dos veículos, fator raramente aplicado nos modelos de otimização do sistema de trans- 
porte. Aliado à utilização da frota heterogênea, a compreensão do comportamento da demanda faz-se totalmente necessária, dada a possibilidade de escalonamento de veículos com diferentes capacidades, buscando sempre aliar qualidade no atendimento, satisfação do passageiro e minimização dos custos de transporte.

Importante ressaltar que a abordagem adotada neste trabalho de geração da tabela de horários integrada ao VTSP consiste em redefinir os horários de algumas viagens de uma tabela ativa. Assim, não é desenvolvida uma tabela de horários inicial para suportar as atividades diárias do transporte público, mas são aprimoradas as tabelas já existentes, garantindo melhor distribuição das viagens e qualidade nos serviços prestados aos passageiros. Modificações sutis na tabela de horários, alterando os horários de partida ou chegada de algumas viagens num intervalo de tempo curto, permitem aumentar drasticamente o espaço de soluções possíveis (Kliewer; Amberg; Amberg, 2012), possibilitando graus de liberdade adicionais ao VTSP. Trabalhos com a perspectiva de integração da formulação e resolução da tabela de horários e do VTPS, tendo como base a TSN, não foram identificados na literatura, o que garante o caráter inédito desta investigação.

Dados disponibilizados pela Associação Nacional dos Transportes Públicos (ANTP, 2012) de que a população dos municípios com mais de 60 mil habitantes realizou, em 2011, 61,3 biIhões de viagens, sendo 17,7 bilhões pelo transporte público, motivam a realização deste estudo, visto a sua importância prática, não somente acadêmica. Ainda, segundo a ANTP (2012), quando as viagens são classificadas por porte dos municípios, percebe-se que a participação do transporte público gira expressivamente em torno de $20 \%$, à exceção das cidades acima de 1 milhão de habitantes, nas quais atinge $36 \%$. Os ônibus públicos no Brasil, no ano de 2011 , destacaram-se como o terceiro meio de transporte mais utilizado, seguindo o transporte a pé e de bicicleta (1ㅇ) e de carros particulares e motocicletas (2), sendo o ônibus o responsável pelo deslocamento de 40 milhões de passageiros diariamente, atendendo $87 \%$ da demanda de transporte público coletivo nacional. A frota de ônibus municipais e metropolitanos no Brasil ultrapassou os 105 mil veículos. Esses dados reforçam a necessidade do desenvolvimento de ferramentas que contribuam no aprimoramento das operações de um dos meios de transporte mais utilizados no país.

A fim de atingir o objetivo proposto, este artigo está divido em três seções, além desta introdução. A seção 2 faz um resgate da literatura, descrevendo as etapas do sistema de transporte público, bem como apresentando artigos correlatos a este, previamente publicados. Na seção 3, é apresentado o método deste estudo, contemplando a modelagem e a definição do problema investigado e a formulação matemática do modelo. Por fim, na seção 4, são apresentados os resultados computacionais, considerando os testes com instâncias reais, advindas do sistema de transporte público da cidade de Santa Maria/RS, e instâncias aleatórias de grande porte.

\section{REVISÃO DE LITERATURA}

Um sistema de transportes é composto pelos passageiros e bens a serem transportados, pelos veículos que efetuam os deslocamentos e pela rede de infraestrutura de transportes que permite a operação do sistema (Manheim, 1980). Assim, no planejamento operacional do transporte público, diferentes etapas são consideradas, de acordo com suas complexidades (Freling; Wagelmans; Paixão, 1999; De Leone; Festa; Marchitto, 2011): a tabela de horários, o escalonamento de veículos, o escalonamento da tripulação e a rotação da tripulação, conforme a Figura 1. 
Figura 1 - Planejamento Operacional dos Sistemas de Transporte

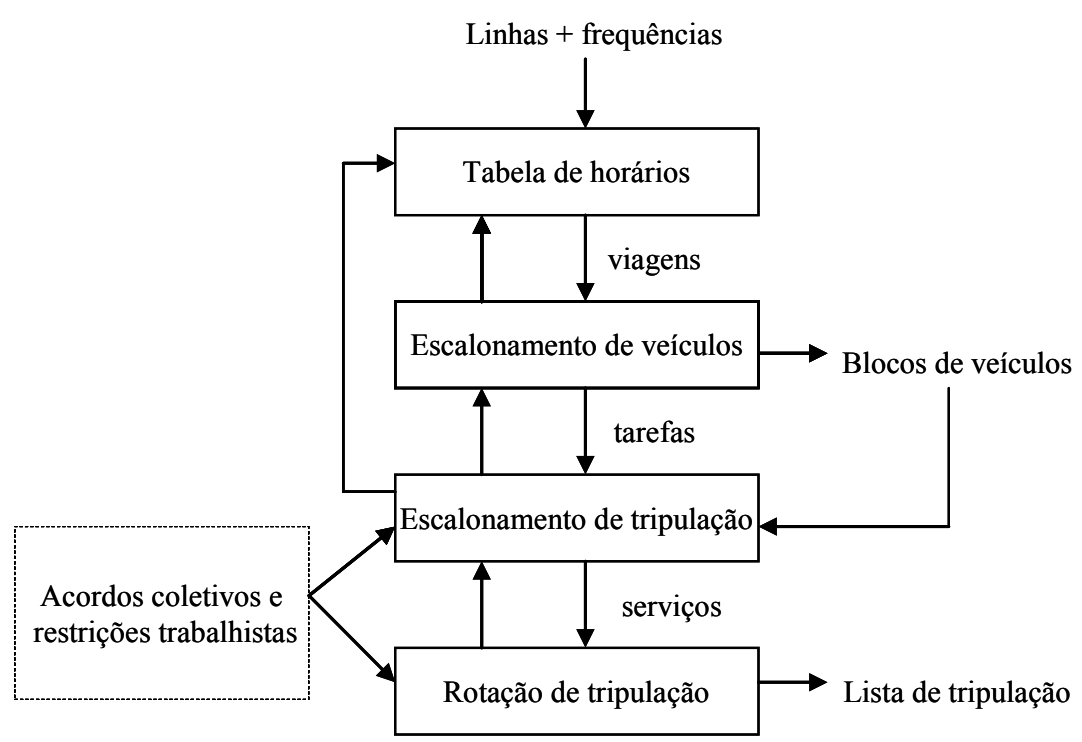

Fonte: elaborada a partir de Freling, Wagelmans e Paixão (1999) e De Leone, Festa e Marchitto (2011).

O serviço de transporte é composto por um conjunto de linhas que correspondem às viagens de ônibus entre dois terminais de uma mesma cidade. Para cada linha, a frequência é determinada pela demanda, pela infraestrutura disponível e pelo requerimento de serviços por parte dos passageiros. Então, uma tabela de horários é construída, resultando em viagens caracterizadas por um local e horário de início e fim. As etapas subsequentes são o escalonamento de veículos e o de tripulação, que correspondem ao direcionamento dos veículos para as viagens e da tripulação para os veículos, respectivamente. No escalonamento dos veículos, cada escala é definida como uma jornada de ônibus que começa em uma garagem e retorna para a mesma garagem. $O$ objetivo é minimizar o custo total dos ônibus usados e do funcionamento. Já no escalonamento da tripulação, tem-se que determinar as jornadas (conjunto de tarefas para um dia de trabalho) para os motoristas a partir de uma dada garagem para cobrir todas as viagens. Por fim, a elaboração da rotação de tripulação é a construção do escalonamento da tripulação para o longo prazo, como um mês ou meio ano.

Entre esses níveis de planejamento, existe um sistema de retroalimentações entre cada atividade e as demais, pois todas estão interligadas, umas mais fortemente do que outras (Prata, 2009). Assim, o planejamento pode ser mais eficiente se suas atividades forem definidas de modo integrado; entretanto, como cada uma das etapas do planejamento é extremamente complexa, é matematicamente inviável considerar o processo todo de uma única vez (Freling; Wagelmans; Paixão, 1999). O planejador deve sempre gerir o trade-off entre os ganhos de efetividade advindos da integração e a complexidade do sistema a ser planejado.

Analisando o contexto da otimização integrada entre as etapas do planejamento do transporte público, Guihaire e Hao (2010) salientam que a abordagem simultânea da modificação da tabela de horários e do escalonamento de veículos é pouco explorada de modo integrado na literatura e pode gerar benefícios ao sistema de transporte público. Realizando um levantamento da literatura, observou-se que a resolução conjunta dos problemas de geração ou modificação da tabela de horários e o escalonamento de veículos pode ocorrer de três modos: 1) pelo acréscimo de janelas de tempo ao modelo de escalonamento de veículos; 2 ) pela otimização conjunta da tabela de horários e do escalonamento dos veículos; ou 3) pela geração iterativa e sequencial da tabela de horários e pelo escalonamento de veículos, em que o resultado do último retroalimenta a otimização da tabela de horários. 
Desaulniers, Lavigne e Soumis (1998) formularam o problema de escalonamento de veículos com múltiplas garagens (MD-VSP) com janelas de tempo como um modelo de fluxo multicommodity não linear inteiro. $\mathrm{O}$ objetivo do problema consiste em alocar a frota para executar todas as tarefas respeitando as restrições de janela de tempo e capacidade dos veículos. Para a resolução, foi utilizada a técnica de geração de colunas incorporada em uma estrutura branch-an$d$-bound. A versão heurística e a versão exata do algoritmo foram testadas em instâncias aleatórias. Os resultados indicaram soluções ótimas para instâncias pequenas e médias e boas soluções heurísticas (limitando o número de nós da árvore a serem explorados) para grandes instâncias (600 tarefas e cinco garagens), em tempo razoável (uma hora).

Kliewer, Bunte e Suhl (2006) também resolvem o MD-VSP com janelas de tempo. Baseando-se em uma rede tempo-espaço, arcos de janela de tempo são adicionados aos arcos já existentes. Esses arcos são caracterizados como "arcos-sombra" dos arcos de serviço originais e representam o deslocamento (adiantamento ou atraso) de uma viagem em alguns poucos minutos. A inserção de arcos de janela de tempo gera um número excessivo de arcos, exigindo tempo de solução computacional extremamente alto. Em virtude disso, Kliewer, Bunte e Suhl (2006) e Kliewer, Amberg e Amberg (2012) desenvolveram técnicas de pré-processamento para evitar a criação de arcos de janela de tempo que não possibilitam a compatibilidade com outras viagens de serviço, quando comparados com o arco de serviço original. Como o modelo é altamente complexo, em Kliewer, Bunte e Suhl (2006) são apresentadas duas heurísticas para ajudar na resolução do escalonamento dos veículos: uma heurística do tipo "trip shortening" e uma heurística de corte.

Em publicação posterior, Kliewer, Amberg e Amberg (2012) adicionam o problema de escalonamento de tripulação ao trabalho apresentado por Kliewer, Bunte e Suhl (2006) e utilizam uma combinação de relaxação lagrangeana e geração de colunas para resolver o problema integrado. As heurísticas desenvolvidas no trabalho de Kliewer, Bunte e Suhl (2006) também são utilizadas como suporte para a resolução do problema. A partir de instâncias disponíveis na literatura, economias no número de veículos e tripulação são encontradas nessa abordagem integrada se comparadas a trabalhos anteriores (Steinzen; Gintner; Suhl, 2010), que não utilizaram janelas de tempo.

Van den Heuvel, van den Akker e Niekerk (2008) propõem duas novas formulações para o VTSP: na primeira, mais de um tipo de veículo pode atender a uma viagem, tornando o problema de difícil resolução; e na segunda, que visa minimizar em partes a complexidade da primeira, permite-se que vários veículos do mesmo tipo atendam à mesma viagem, sendo que os ônibus são distribuídos uniformemente no tempo. Se, por exemplo, há uma viagem a cada hora, e serão necessários dois ônibus de um determinado tipo, um veículo sairia do terminal a cada meia-hora. Isso é obtido através da inclusão de viagens de serviço adicionais na rede tempo-espaço. Esse problema, ao flexibilizar a frota, aumenta significativamente a complexidade de resolução computacional se comparado ao VSP para um único tipo de veículo. Após encontrado o escalonamento adequado, os autores realizam pequenas modificações na tabela de horários previamente utilizada, através da aplicação da meta-heurísitica Simulated Annealing, com o objetivo de meIhor adequar os veículos à demanda existente para cada viagem. São testadas instâncias reais da companhia de ônibus holandesa Connexxion. Os resultados desse problema, integrando a frota heterogênea e as modificações na tabela de horários, indicam economias de até $8 \%$ se comparados aos resultados do problema resolvido por Kliewer, Mellouli e Suhl (2006), no qual não havia sincronização com a tabela de horários. Entre os trabalhos encontrados na literatura, o de van den Heuvel, van den Akker e Niekerk (2008) é o que apresenta maiores similaridades com a nova abordagem proposta neste artigo. Entretanto, nesta nova abordagem, a modificação da tabela de horários e o escalonamento da frota heterogênea são realizados de modo simultâneo, a partir da 
formulação matemática integrada desses problemas, não necessitando de pós-processamento para ajustar os horários de partida das viagens.

Recentemente, Ceder, Hassold e Dano (2013) desenvolveram uma metodologia multiobjetivo para criar horários de ônibus usando vários tamanhos de veículos, com dois objetivos realizados simultaneamente: primeiro, minimizar o tempo de espera dos passageiros nos terminais e, segundo, minimizar o desvio de número de passageiros observados e desejados em cada ônibus. Para tanto, duas heurísticas foram desenvolvidas e testadas em instâncias de Auckland, Nova Zelândia, demonstrando bons resultados em termos de redução de espera dos passageiros e melhor aproveitamento dos veículos. Entretanto, o modelo só pode ser aplicado a linhas de ônibus individuais, não podendo considerar rotas interligadas ou um conjunto de linhas. Para tanto, os autores sugerem que pesquisas futuras devem ser realizadas nessa direção, sincronizando o escalonamento de veículos de diferentes tipos e a geração da tabela de horários, pois esse tipo de análise combinada "irá demonstrar como minimizar o custo operacional, melhorando, simultaneamente, a qualidade dos serviços ao permitir o escalonamento de vários tamanhos de ônibus" (CEDER; HASSOLD; DANO, 2013, p. 216). É nessa direção, de minimizar custos operacionais e atender à demanda de passageiros com qualidade, que se desenvolve o modelo apresentado neste artigo.

\section{METODOLOGIA}

Esta seção trata dos aspectos metodológicos que nortearam o desenvolvimento deste estudo. Primeiramente, apresenta-se a modelagem da rede TSN utilizada no problema de otimização investigado. Na sequência, é definido o problema e apresentada a sua formulação matemática.

\subsection{Modelagem do problema}

O problema de escalonamento de uma frota homogênea de veículos para um único depósito é representado através de um modelo de fluxo em rede e expresso por uma rede tempo -espaço. A estrutura da TSN foi primeiramente aplicada para o VTSP por Kliewer, Mellouli e Suhl (2002), sendo utilizada, posteriormente, por Kliewer, Mellouli e Suhl (2006), Kliewer, Bunte e Suhl (2006b) e Kliewer, Amberg e Amberg (2012). A principal vantagem dessa estrutura é a diminuição do número de variáveis e restrições, se comparada com a rede baseada em conexões, visto que nela evita-se o inconveniente de consideração explícita de todas as conexões possíveis entre viagens compatíveis, garantindo todos os escalonamentos viáveis de veículos.

$\mathrm{Na}$ TSN, cada viagem é representada por dois nós conectados por um arco, sendo definida por um grafo $G=(N, A)$, em que $N$ representa o conjunto de nós e $A$ o conjunto de arcos. Cada nó $n \in N$ representa um local específico em um tempo determinado, e devem ser definidos em função de $l$ o local de início ou fim de uma viagem e de $t$ o tempo de início ou fim de uma viagem. Os locais de início e fim de uma viagem podem ser um terminal $s \in S$ ou uma garagem $d \in D$. Logo, o conjunto de nós $N=\{(l, t) \mid l \in S \cup D \wedge t \in T\}$, em que $T$ representa o horizonte de planejamento.

Cada arco $a \in A$ da TSN corresponde à transição no tempo e, provavelmente, no espaço. O conjunto $A$ é composto por seis subconjuntos, $A=\left\{A^{\text {se }}, A^{\text {wait }}, A^{\text {dh }}, A^{\text {pin }}, A^{\text {pout }}, A^{c}\right\}$, sendo cada um desses arcos definido como:

$A^{\text {se }}$ é o conjunto dos arcos de serviço, utilizados para conectar dois nós compatíveis da rede, localizados em diferentes terminais, com o objetivo de atender à demanda. Cada arco 
de serviço é definido em função de um terminal e um horário de início de uma viagem $\left(l_{i}, t_{i}\right)$ e um terminal e um horário de fim de uma viagem $\left(l_{j}, t_{j}\right)$. Duas viagens $i$ e $i$ são compatíveis se puderem ser cobertas pelo mesmo veículo.

$A^{\text {wait }}$ é o conjunto dos arcos de espera, representando transições no tempo, mas não no espaço, ou seja, um arco de espera indica que o veículo está parado em um terminal ou na garagem.

$A^{d h}$ é o conjunto dos arcos deadhead (dh), utilizados para conectar dois nós que sejam compatíveis e que estejam localizados em diferentes terminais. Nesta situação, o veículo deslocase vazio, sem atender à demanda durante o trajeto.

$A^{\text {pin }}$ é o conjunto dos arcos pull-in, empregados quando um veículo parte da garagem em direção a um terminal. Assim, um arco pull-in conecta um nó da garagem a um nó em um terminal. $A^{\text {pout }}$ é o conjunto dos arcos pull-out, inseridos quando um veículo parte de um terminal em direção à garagem. Assim, um arco pull-out conecta um nó de um terminal a um nó na garagem.

$A^{c}$ é o conjunto dos arcos de circulação. Como a TSN é desenhada para o período de um dia de trabalho, cada unidade de fluxo do último para o primeiro nó da garagem define o escalonamento diário de um veículo e corresponde a um arco de circulação. A linha de tempo da garagem é apresentada em forma cíclica para forçar a circulação na rede, necessidade imposta pelas restrições de fluxo.

A fim de ilustrar uma TSN, apresenta-se a Figura 2.

Figura 2 - Rede tempo-espaço com seis viagens, uma garagem e três terminais
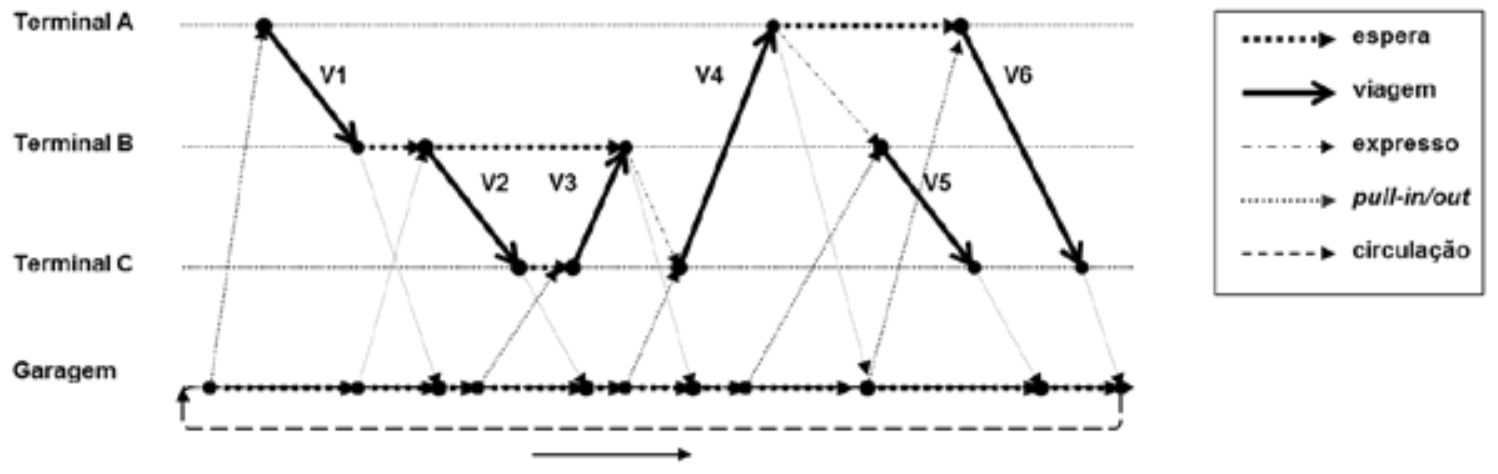

Fonte: adaptada de Steinzen, Gintner e Suhl (2010).

Na representação da Figura 2, percebe-se a inexistência de um arco de espera entre as viagens V3 e V5, pois assume-se que é mais barato o veículo voltar à garagem se o tempo ocioso no terminal for superior a um limite estabelecido. Entretanto, se esse aspecto não for levado em consideração, pode-se acrescentar um arco de espera entre essas viagens. Já o veículo que realizou a viagem $\mathrm{V} 4$, por exemplo, assume três diferentes possibilidades ao término da viagem: voltar à garagem, aguardar no terminal $A$ para realizar a viagem $V 6$ ou realizar uma viagem expressa até o terminal $\mathrm{B}$ para realizar a viagem $\mathrm{V} 5$.

A base da TSN é explorar a propriedade de transitividade de conjuntos ordenados, indicando que, para viagens $p, q$ e $v$, a seguinte conclusão é aplicada (Bunte; Kliewer, 2009):

$(p R q) \wedge(q R v) \rightarrow p R v$, onde $R$ é uma relação binária

A aplicação dessa propriedade à TSN é facilmente visualizada quando se aplicam pré -processamentos para a redução no número de arcos da rede. Esses pré-processamentos têm impacto significativo no número de variáveis e restrições da formulação matemática (Kliewer; Mellouli; Suhl, 2002, 2006; van den Heuvel; van den Akker; Niekerk, 2008; Visentini et al., 2013). 
Para considerar a frota heterogênea, deve-se construir uma estrutura de rede para cada tipo de veículo, conforme explicitado na Figura 3.

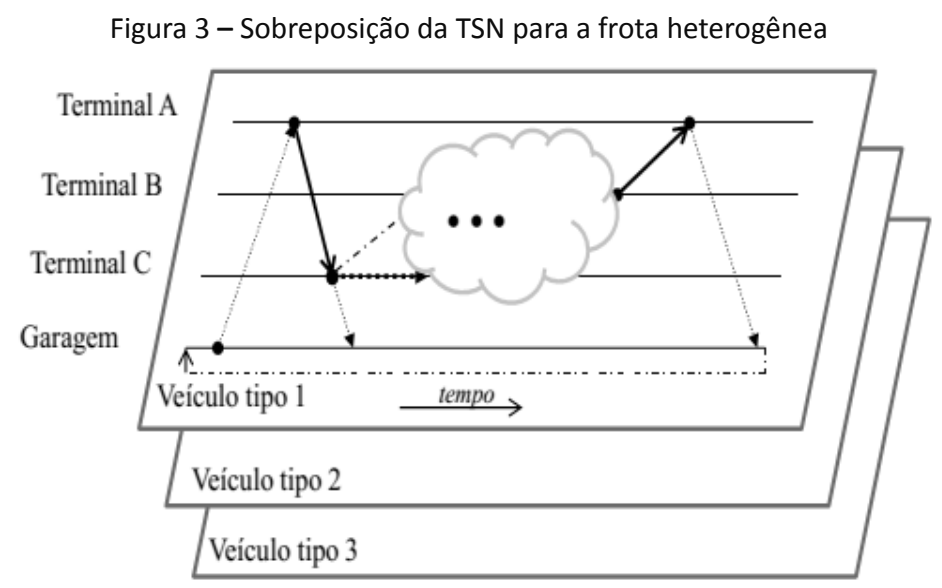

Fonte: adaptada de Bunte e Kliewer (2009).

Essa sobreposição de redes por tipo de veículo possibilita que uma mesma viagem de serviço esteja em várias camadas, não sendo possível resolver essas camadas de forma independente, caracterizando um problema de rede com fluxo multicommodity. Assim, se houver dois ou mais veículos, o problema torna-se NP-difícil (Bertossi; Carraresi; Gallo, 1987; van den Heuvel; van den Akker; Niekerk, 2008).

\subsection{Definição do problema e formulação matemática do modelo}

O problema integrado de geração da tabela de horários e escalonamento de veículos com frota heterogênea combina as duas primeiras etapas do planejamento operacional do transporte público. Dado um conjunto de viagens com um horizonte de planejamento fixo, propõe-se minimizar o custo do escalonamento e/ou o número total de veículos. A partir da ampliação do modelo VTSP, apresentado por van den Heuvel, van den Akker e Niekerk (2008), o modelo proposto neste artigo, chamado de VTSP-SCT (Vehicle Type Scheduling Problem with Sequential Change of Timetable), visa minimizar o número total de veículos, considerando a frota heterogênea, e, simultaneamente, readequar a tabela de horários vigente. Dadas as diferentes capacidades dos veículos, o modelo (a) possibilita direcionar o (tipo de) veículo mais adequado para cada viagem, (b) readequar a tabela de horários à demanda histórica e (c) minimizar os custos operacionais. Para que seja possível ajustar a tabela de horários, define-se um intervalo de tempo $\Delta t$ em minutos e analisam-se todas as viagens de serviço que possuem horários de partida próximos, com uma diferença máxima igual ao valor de $\Delta t$. Esse intervalo de tempo é de poucos minutos, a fim de minimizar os efeitos sentidos pelos passageiros na variação dos horários de ônibus. As viagens analisadas dentro do intervalo $\Delta t$ devem possuir igual terminal de partida e destino, para que se possam identificar possibilidades de readequação de horários e tipos de veículos para atender à demanda total.

Para identificar quais viagens podem ser agrupadas dentro do intervalo $\Delta t$, é necessário considerar as viagens em sequência, a partir do horário da primeira viagem do dia em direção ao horário da última viagem. Na Figura 4, é apresentado o algoritmo que descreve a rotina de geração de intervalos $k \in K$ a partir da TSN. As viagens de serviço $\left(A_{k}^{s e}\right)$ na TSN são ordenadas pelo seu horário de partida em cada terminal $s$, sendo $t_{0 s}$ o horário de partida da primeira viagem do dia do horizonte de planejamento da terminal $s, t_{E s}$ o tempo de partida da última viagem da 
terminal $s$ e $A_{k}^{s e}$ o conjunto dos arcos de serviço pertencentes ao intervalo $k \in K$. Consideramse os conjuntos $A_{k}^{s e}, k \in K$ mutuamente exclusivos.

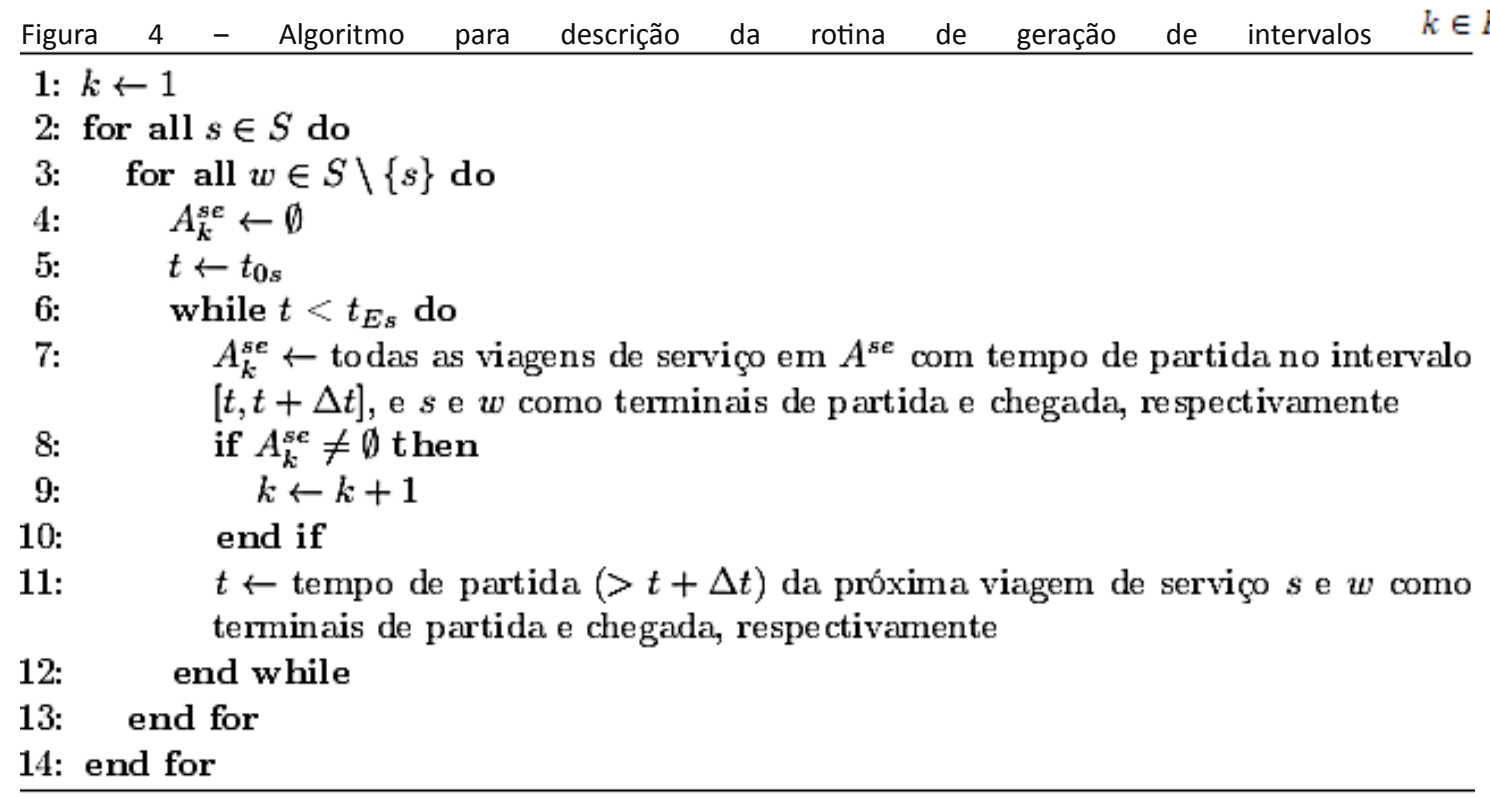

Fonte: elaborada pelos autores.

A Figura 5 representa o agrupamento de viagens no modelo VTSP-SCT, considerando os horários de partida e chegada de viagens com mesmo terminal de origem (Terminal A) e destino (Terminal B).

Figura 5 - Agrupamento sequencial de viagens no modelo VTSP-SCT

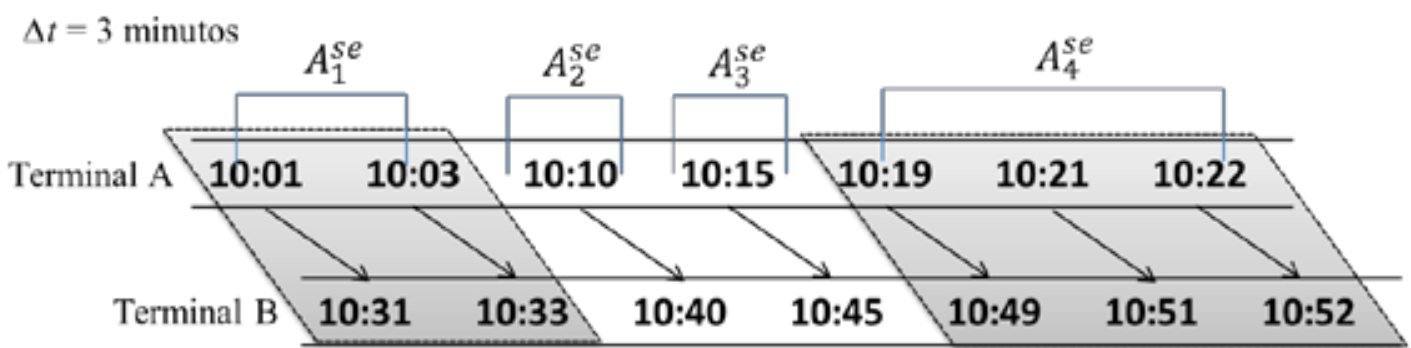

Fonte: elaborada pelos autores.

Considerando-se $\Delta t=3$ minutos, tem-se para o intervalo de índice $k=1$ o conjunto $A_{1}^{s e}$ formado pelas viagens que partem nos instantes 10:01 e 10:03 do terminal A, os intervalos de índice $k=2$ e $k=3$ compostos por apenas uma viagem cada e, para o intervalo de índice $k=4$, o conjunto $A_{4}^{s e}$, composto pelas viagens com partida às 10:19, 10:21 e 10:22. A análise do modelo VTSP-SCT recai especificamente sobre os intervalos de índice $k=1$ e $k=4$, identificando se há a possibilidade de realocação da demanda total desses intervalos entre as viagens que o compõem. Se, por exemplo, for possível eliminar uma ou duas viagens do intervalo de índice $k=4$, o(s) veículo(s) que realizará(ão) as outras viagens deverá(ão) comportar a demanda de todas as viagens desse intervalo. A distribuição da demanda de um intervalo será realizada de acordo com a capacidade dos veículos que atenderão ao intervalo. Assim, pode-se ter um único 
veículo aue comporte a demanda total das três viagens (10:19, 10:21 e 10:22) do intervalo de índice $k=4$, ou talvez sejam necessários dois veículos, sendo que a demanda total das viagens será dividida entre eles.

O modelo VTSP-SCT pode ser formulado conforme segue. Seja $K$ o conjunto dos intervalos de índice $k, A$ o conjunto de todos os arrns da rede, $A_{k}^{s e}$ o conjunto das viagens de servico pertencentes ao intervalo de índice $k \in K, p_{f}$ a capacidade do veículo do tipo $f \in F$ e $P_{k}$ a demanda total esperada para o intervalo de índice $k \in K$, o modelo VTSP-SCT pode ser representado por:

$$
\begin{aligned}
& V T S P-S C T: \sum_{(i, j) \in A} \sum_{f \in F} c_{i j f} x_{i j f} \\
& \sum_{(i, j) \in A} x_{i j f}-\sum_{(j, l) \in A} x_{j l f}=0 \\
& \sum_{(i, j) \in A_{k}^{s e}} \sum_{f \in F} p_{f} x_{i j f} \geq P_{k} \\
& \sum_{f \in F} x_{i j f} \leq 1 \\
& x_{i j f} \in \mathbb{N}
\end{aligned}
$$

$$
\begin{aligned}
& \forall j \in N, \forall f \in F \\
& \forall k \in K \\
& \forall(i, j) \in A^{s e} \\
& \forall(i, j) \in A, \forall f \in F
\end{aligned}
$$

A função objetivo (1) minimiza o custo total dos veículos. As restrições (2) referem-se às propriedades de fluxo da rede, e as restrições (3) garantem que a capacidade dos veículos que realizarão as viagens inseridas no intervalo de índice ${ }^{k}$ atenda à demanda total do intervalo. A partir dessas duas restrições, a tabela de horários e o escalonamento dos veículos são integrados, desde que o número de veículos necessários para atender à demanda seja menor do que o número de arcos de serviço no intervalo $k \in K$. Assim, algumas viagens podem ser eliminadas da tabela de horários. As restrições (4) asseguram que todas as viagens serão operadas, no máximo, uma vez por um único veículo de determinado tipo. Combinando as restrições (2) e (4), pode-se concluir que cada arco de serviço será atendido por, no máximo, um veículo, mas em todos os outros arcoc da TSN mais de um veículo poderá transitar. As restrições (5) referem-se ao domínio da variável ${ }^{x_{i j f}}$.

\section{RESULTADOS COMPUTACIONAIS}

O modelo VTSP-SCT foi implementado na linguagem de programação $\mathrm{C}++$, e os testes computacionais, executados em uma máquina com processador Intel ${ }^{\circ} \mathrm{Xeon}^{\circ} \mathrm{CPU}$ E5-1603 com $2.8 \mathrm{GHz}$ e $16 \mathrm{~GB}$ RAM. O resolvedor de Programação Linear utilizado foi o IBM $^{\circ}$ ILOG $^{\circ} \mathrm{CPLEX}^{\circ}$ Optimization Studio V12.5, com tempo limite escolhido arbitrariamente de 7.200 segundos. Os testes foram realizados com instâncias reais, advindas do sistema de transporte público da cidade de Santa Maria/RS, e instâncias aleatórias de grande porte, permitindo validar a robustez do modelo na resolução de problemas com um grande número de variáveis e restrições. Na Tabela 1, são apresentadas as características das instâncias reais e aleatórias, com as respectivas origens (Origem), número de viagens (\#Viagens), número de terminais (\#Terminais) e número de veículos utilizados na prática para as instâncias reais (\#Veículos). Para cada uma das dez configurações das instâncias aleatórias, foram geradas cinco instâncias e realizada a média das mesmas para a 
obtenção dos resultados de cada configuração. A partir de uma análise do comportamento da demanda no sistema de transporte público de Santa Maria, geraram-se as instâncias totalmente aleatórias, com número mínimo de passageiros igual a 20 e número máximo igual a 110.

Tabela 1 - Características das instâncias testadas

\begin{tabular}{ccccc}
\hline Configuração & Origem & \#Viagem & \#Terminais & \#Veículos \\
\hline 60_8 & Real & 60 & 8 & 15 \\
469_9 & Real & 469 & 9 & 43 \\
530_16 & Real & 530 & 16 & 58 \\
1000_10 & Aleatória & 1.000 & 10 & - \\
1000_23 & Aleatória & 1.000 & 23 & - \\
2000_10 & Aleatória & 2.000 & 10 & - \\
2000_23 & Aleatória & 2.000 & 23 & - \\
3000_10 & Aleatória & 3.000 & 10 & - \\
3000_23 & Aleatória & 3.000 & 23 & - \\
4000_10 & Aleatória & 4.000 & 10 & - \\
4000_23 & Aleatória & 4.000 & 23 & - \\
5000_10 & Aleatória & 5.000 & 10 & - \\
5000_23 & Aleatória & 5.000 & 23 & - \\
\hline
\end{tabular}

Fonte: elaborada pelos autores.

A frota heterogênea considerada é referente a três tipos de veículos: o do tipo $A$, com capacidade de 141 lugares; o do tipo B, com 100 lugares; e o do tipo C, com 83 lugares. Para cada tipo de veículo, foi definido um fator multiplicador dos custos, diretamente proporcional à capacidade do ônibus. Ainda, definiu-se um alto custo fixo $10^{6}$, a fim de minimizar o número total de veículos; dessa forma, os valores exibidos para a função objetivo (F.O) dos modelos serão elevados, pois serão balizados por essa ordem de grandeza.

\subsection{Testes com instâncias reais}

Estes resultados objetivam comparar o número de veículos escalonados a partir da aplicação do modelo VTSP-SCT com os utilizados na prática e os escalonados pelo modelo VTSP (sem modificação da tabela de horários). Quando se aplica o modelo VTSP-SCT às instâncias reais, busca-se definir algumas viagens que poderiam ser eliminadas da tabela de horários vigente (\#Viagens agrupadas), visto que estão disponíveis veículos com diferentes capacidades, minimizando a frota necessária para atender aos usuários. Na Tabela 2, são apresentados os resultados computacionais para essas comparações, considerando-se intervalos de tempo $(\Delta t)$ de 1,2 e 3 minutos.

Tabela 2 - Resultados computacionais para as instâncias reais

\begin{tabular}{|c|c|c|c|c|c|c|c|c|c|}
\hline \multirow{2}{*}{ Instância } & \multirow{2}{*}{ Modelo } & \multirow{2}{*}{$\Delta \mathrm{t}$} & \multirow{2}{*}{ F.O. } & \multirow{2}{*}{$\begin{array}{l}\text { Tempo } \\
\text { (seg.) }\end{array}$} & \multicolumn{4}{|c|}{ \#Veículos } & \multirow{2}{*}{$\begin{array}{c}\text { \#Viagens } \\
\text { agrupadas }\end{array}$} \\
\hline & & & & & $A$ & $B$ & c & Total & \\
\hline \multirow{4}{*}{$60 \_8$} & VTSP & 0 & $1,412 \mathrm{E}+11$ & 0,04 & 1 & 2 & 10 & 13 & 0 \\
\hline & & 1 & $1,412 \mathrm{E}+11$ & 0,03 & 1 & 2 & 10 & 13 & 0 \\
\hline & VTPS-SCT & 2 & $1,412 \mathrm{E}+11$ & 0,02 & 1 & 2 & 10 & 13 & 0 \\
\hline & & 3 & $1,362 E+11$ & 0,03 & 2 & 1 & 9 & 12 & 1 \\
\hline
\end{tabular}




\begin{tabular}{cccccccccc}
\hline & VTSP & 0 & $4,166 \mathrm{E}+11$ & 1,08 & 0 & 3 & 38 & 41 & 0 \\
\multirow{2}{*}{ 469_9 } & & 1 & $4,136 \mathrm{E}+11$ & 1,10 & 1 & 3 & 36 & 40 & 5 \\
& \multirow{2}{*}{ VTPS-SCT } & 2 & $3,876 \mathrm{E}+11$ & 1,91 & 1 & 5 & 31 & 37 & 16 \\
& & 3 & $3,766 \mathrm{E}+11$ & 2,72 & 4 & 4 & 26 & 34 & 24 \\
\hline \multirow{2}{*}{$530 \_16$} & VTSP & 0 & $5,278 \mathrm{E}+11$ & 1,67 & 1 & 5 & 45 & 51 & 0 \\
& \multirow{2}{*}{ VTPS-SCT } & 1 & $5,217 \mathrm{E}+11$ & 2,33 & 3 & 5 & 41 & 49 & 13 \\
& & 2 & $4,907 \mathrm{E}+11$ & 3,19 & 4 & 6 & 35 & 45 & 25 \\
\hline
\end{tabular}

Fonte: elaborada pelos autores.

Considerando-se a instância de menor porte (60_8), pôde-se diminuir de 13 para 12 o número de veículos necessários para atender à demanda, se comparados os modelos VTSP e VTSP-SCT com intervalo $\Delta t=3$ minutos, respectivamente. Para esse mesmo $\Delta t$, quando analisada a redução do número de veículos frente ao número de veículos utilizados na prática, tem-se três veículos a menos, sendo necessário o agrupamento de somente uma viagem. Para a instância 469_9, obtém-se redução ainda maior no número de veículos quando aplicado $\Delta t=3$ minutos, passando de 41 veículos do VTSP para 34 no VTSP-SCT, sendo necessário o agrupamento de 24 viagens. Consequentemente, na obtenção deste resultado, foi necessário aumentar a quantidade de veículos do tipo A (articulados), para comportar a demanda das viagens agrupadas. Comparando-se com os veículos escalonados na prática, pôde-se diminuir nove ônibus ao utilizar a frota heterogênea, garantindo o atendimento da demanda historicamente registrada. Por fim, para a instância 530_16, nota-se a maior economia de veículos, sendo estes reduzidos de 51, com o VTSP, para 40, com o VTSP-SCT, considerando $\Delta t=3$ minutos. Para tanto, agruparam-se 45 viagens, sendo necessários nove ônibus do tipo $A$, cinco do tipo $B$ e 26 do tipo $C$ para atender à demanda. Ainda, comparando-se a necessidade de veículos no contexto real, pode-se reduzir em até 18 veículos a frota vigente ao considerar $\Delta t=3$ minutos.

Conforme os resultados apresentados na Tabela 2, constata-se que a aplicação do modelo VTSP-SCT proporciona, além da redução dos custos, flexibilidade ao gestor na escolha de qual a melhor configuração de escalonamento a utilizar (deltas), dados os recursos disponíveis. A análise de cenários possibilitada pela aplicação desse modelo de otimização tende a contribuir na melhoria da tomada de decisão das empresas de transporte público, tanto no sentido de renovação da frota quanto na melhor distribuição dos veículos por linhas de ônibus de acordo com a demanda.

\subsection{Testes com instâncias aleatórias}

Os testes computacionais do modelo VTSP-SCT para as instâncias aleatórias são apresentados na Tabela 3 e visam validar o modelo e identificar o seu comportamento para instâncias de grande porte. 
Tabela 3 - Resultados computacionais para as médias das instâncias aleatórias

\begin{tabular}{|c|c|c|c|c|c|c|c|c|c|}
\hline \multirow{2}{*}{ Instância } & \multirow{2}{*}{ Modelo } & \multirow{2}{*}{$\Delta t$} & \multirow{2}{*}{ F.O. } & \multirow{2}{*}{$\begin{array}{c}\text { Tempo } \\
\text { (seg.) }\end{array}$} & \multicolumn{4}{|c|}{ \#Veículos } & \multirow{2}{*}{$\begin{array}{l}\text { \#Viagens } \\
\text { agrupadas }\end{array}$} \\
\hline & & & & & $A$ & $B$ & $C$ & Total & \\
\hline \multirow{4}{*}{ 1000_10 } & VTSP & 0 & $6,820 E+11$ & 14,25 & 11,0 & 17,2 & 28,8 & 57,0 & 0,0 \\
\hline & & 1 & $6,760 E+11$ & 14,40 & 11,0 & 17,2 & 28,2 & 56,4 & 8,0 \\
\hline & VTSP-SCT & 2 & $6,716 \mathrm{E}+11$ & 16,14 & 11,0 & 17,0 & 28,0 & 56,2 & 14,4 \\
\hline & & 3 & $6,636 \mathrm{E}+11$ & 14,87 & 11,0 & 17,0 & 27,2 & 54,6 & 20,0 \\
\hline \multirow{4}{*}{ 1000_23 } & VTSP & 0 & $7,173 E+11$ & 41,48 & 9,8 & 20,2 & 30,8 & 60,8 & 0,0 \\
\hline & & 1 & $7,153 E+11$ & 35,66 & 9,8 & 20,2 & 30,6 & 60,6 & 2,2 \\
\hline & VTSP-SCT & 2 & $7,153 E+11$ & 34,47 & 9,8 & 20,2 & 30,6 & 60,6 & 3,6 \\
\hline & & 3 & $7,133 E+11$ & 36,36 & 9,8 & 20,2 & 30,4 & 60,4 & 5,4 \\
\hline \multirow{4}{*}{ 2000_10 } & VTSP & 0 & $1,122 E+12$ & 62,79 & 17,6 & 33,2 & 42,4 & 93,2 & 0,0 \\
\hline & & 1 & $1,096 \mathrm{E}+12$ & 68,74 & 17,6 & 34,0 & 38,8 & 90,4 & 23,0 \\
\hline & VTSP-SCT & 2 & $1,086 \mathrm{E}+12$ & 73,78 & 17,6 & 34,2 & 37,6 & 89,4 & 47,6 \\
\hline & & 3 & $1,074 \mathrm{E}+12$ & 79,97 & 17,6 & 34,2 & 36,4 & 88,2 & 67,6 \\
\hline \multirow{4}{*}{ 2000_23 } & VTSP & 0 & $1,255 E+12$ & 171,94 & 21,0 & 38,6 & 43,4 & 103,0 & 0,0 \\
\hline & & 1 & $1,253 E+12$ & 167,67 & 21,0 & 38,6 & 43,2 & 102,8 & 4,6 \\
\hline & VTSP-SCT & 2 & $1,253 E+12$ & 173,24 & 21,0 & 38,6 & 43,2 & 102,8 & 8,8 \\
\hline & & 3 & $1,253 E+12$ & 186,14 & 21,0 & 38,6 & 43,2 & 102,8 & 12,6 \\
\hline \multirow{4}{*}{ 3000_10 } & VTSP & 0 & $1,601 E+12$ & 139,70 & 25,2 & 51,0 & 56,0 & 132,2 & 0,0 \\
\hline & & 1 & $1,569 \mathrm{E}+12$ & 162,62 & 25,4 & 51,2 & 52,2 & 128,8 & 48,4 \\
\hline & VTSP-SCT & 2 & $1,554 \mathrm{E}+12$ & 155,85 & 26,0 & 51,0 & 50,0 & 127,0 & 96,4 \\
\hline & & 3 & $1,544 \mathrm{E}+12$ & 204,67 & 26,2 & 51,0 & 48,6 & 125,8 & 139,8 \\
\hline \multirow{4}{*}{ 3000_23 } & VTSP & 0 & $1,754 \mathrm{E}+12$ & 393,86 & 27,4 & 55,8 & 61,8 & 145,0 & 0,0 \\
\hline & & 1 & $1,749 \mathrm{E}+12$ & 440,95 & 27,4 & 57,2 & 59,6 & 144,2 & 10,0 \\
\hline & VTSP-SCT & 2 & $1,745 E+12$ & 451,95 & 27,6 & 55,6 & 60,8 & 144,0 & 21,0 \\
\hline & & 3 & $1,743 E+12$ & 449,14 & 27,6 & 55,4 & 60,8 & 143,8 & 32,6 \\
\hline \multirow{4}{*}{ 4000_10 } & VTSP & 0 & $2,022 E+12$ & 288,80 & 31,4 & 57,6 & 79,6 & 168,6 & 0,0 \\
\hline & & 1 & $1,975 E+12$ & 319,66 & 30,4 & 56,8 & 77,6 & 164,8 & 86,4 \\
\hline & VTSP-SCT & 2 & $1,913 E+12$ & 336,31 & 31,6 & 58,6 & 67,2 & 157,4 & 168,4 \\
\hline & & 3 & $1,885 E+12$ & 405,62 & 32,0 & 58,8 & 63,2 & 154,0 & 250,6 \\
\hline \multirow{4}{*}{ 4000_23 } & VTSP & 0 & $2,175 \mathrm{E}+12$ & 851,65 & 33,0 & 65,8 & 82,4 & 181,2 & 0,0 \\
\hline & & 1 & $2,165 E+12$ & 852,81 & 33,0 & 65,8 & 81,4 & 180,2 & 18,4 \\
\hline & VTSP-SCT & 2 & $2,156 \mathrm{E}+12$ & 827,01 & 33,0 & 65,8 & 80,0 & 178,8 & 35,2 \\
\hline & & 3 & $2,148 \mathrm{E}+12$ & 829,84 & 33,0 & 66,0 & 79,4 & 178,4 & 52,6 \\
\hline \multirow{4}{*}{ 5000_10 } & VTSP & 0 & $2,438 \mathrm{E}+12$ & 391,54 & 39,2 & 75,6 & 86,4 & 201,2 & 0,0 \\
\hline & & 1 & $2,379 E+12$ & 480,30 & 39,6 & 75,8 & 79,6 & 195,0 & 132,6 \\
\hline & VTSP-SCT & 2 & $2,318 \mathrm{E}+12$ & 551,44 & 40,2 & 75,8 & 72,0 & 188,0 & 257,6 \\
\hline & & 3 & $2,306 \mathrm{E}+12$ & 961,87 & 40,6 & 76,4 & 69,8 & 186,8 & 379,8 \\
\hline \multirow{4}{*}{ 5000_23 } & VTSP & 0 & $2,689 \mathrm{E}+12$ & 1277,44 & 43,8 & 80,6 & 97,6 & 222,0 & 0,0 \\
\hline & & 1 & $2,672 E+12$ & 1398,42 & 44,0 & 80,4 & 95,8 & 220,2 & 24,8 \\
\hline & VTSP-SCT & 2 & $2,654 \mathrm{E}+12$ & 1501,14 & 44,0 & 80,4 & 94,0 & 218,4 & 53,4 \\
\hline & & 3 & $2,635 E+12$ & 1483,89 & 43,8 & 80,6 & 92,0 & 216,4 & 76,4 \\
\hline
\end{tabular}

Fonte: elaborada pelos autores.

Todas as instâncias foram resolvidas na otimalidade em tempo bastante inferior ao limite estabelecido (7.200 segundos) e possibilitaram resultados, em termos de número médio de veículos, inferiores aos encontrados no modelo VTSP. Além disso, é perceptível que, quanto maior o número de terminais, menor a quantidade de viagens agrupadas, tendo em vista que há uma dispersão maior entre os itinerários das viagens, impossibilitando a maioria dos agrupamentos. 
Tendo em vista a grande quantidade de resultados apresentados na Tabela 3, esta análise será realizada de modo a discutir os resultados mais relevantes. De modo geral, as instâncias de mil viagens foram as que apresentaram menor redução no número de veículos - comparando-se todas as instâncias aleatórias testadas -, agrupando quantidade não tão elevada de viagens. Esse resultado é esperado, tendo em vista que, comparada com as instâncias com maior densidade, nesta a distribuição das viagens é mais afastada, dificultando o agrupamento em intervalos curtos de $\Delta t$.

Já a solução das instâncias 4000_10 e 5000_10 indica um comportamento típico de baixa demanda para as viagens. Isso pode ser visualizado a partir do aumento na quantidade de viagens agrupadas quando se acrescenta uma unidade de tempo ao $\Delta t$, aliado ao acréscimo de veículos do tipo $\mathrm{A}$ (maior capacidade) escalonados e à redução dos veículos do tipo $\mathrm{C}$ (menor capacidade) necessários para atender à demanda. Baixas demandas, ainda, costumam refletir em um melhor aproveitamento dos veículos quando utilizada a frota heterogênea aliada aos ajustes da tabela de horários, tanto que, nesta instância, reduziu-se a frota significativamente ao se realizar modificações de poucos minutos na tabela de horários. Ainda, para essas instâncias, nota-se a maior economia de veículos na comparação entre o modelo VTSP-SCT e o VTSP. Quando aplicado $\Delta t=3$ minutos, obtêm-se, em média, 15 veículos a menos no modelo VTSP-SCT.

Por fim, na instância 5000_23, observam-se os resultados computacionais com maior tempo total de resolução, em média. Este resultado é esperado, tendo em vista o alto número de viagens e terminais desta instância. Além disso, pôde-se visualizar um comportamento bastante comum do modelo VTSP-SCT: aumentar o tempo de resolução para $\Delta t$ maiores, pois há mais possibilidades de agrupamento nesses intervalos. Mesmo os tempos computacionais acima de 3.600 segundos não inviabilizam a aplicação prática deste modelo, tendo em vista o custo-benefício que se obtém ao utilizá-lo, possibilitando a redução de um grande número de veículos. Além disso, considerando-se que muitos gestores ainda realizam o escalonamento dos veículos manualmente, tarefa bastante demorada, o período de uma ou duas horas para a resolução computacional de uma instância torna-se razoável.

De modo geral, o modelo VTSP-SCT possibilitou a redução no número de veículos sem necessitar de muito tempo de resolução computacional para as instâncias de grande porte, o que confirma a sua aplicabilidade no planejamento dos sistemas de transporte público.

\section{CONSIDERAÇÕES FINAIS}

O desenvolvimento de técnicas de pesquisa operacional que contribuam para o melhor planejamento dos sistemas de transporte público tende a minimizar problemas oriundos do tráfego urbano, bem como auxiliar no melhor atendimento à população. Baseando-se nessa perspectiva, bem como a partir de necessidades gerenciais observadas na prática de empresas de transporte público, neste trabalho foi proposto um novo modelo para a resolução do problema integrado de geração de tabela de horários e escalonamento de veículos com frota heterogênea. $O$ objetivo desse modelo é minimizar o número de veículos e redefinir a tabela de horários a partir do agrupamento de algumas viagens, conforme a demanda de passageiros e a capacidade dos diferentes tipos de veículos disponíveis. A integração dessas duas etapas tem sido reportada na literatura como de grande valia para um melhor planejamento. Ademais, ao considerar a frota heterogênea, imprimese maior realidade à formulação dos problemas, tendo em vista que a utilização de ônibus urbanos de variados tipos é a prática na maioria das cidades ao redor do mundo.

Para modelar o problema tratado, utilizou-se a TSN, tradicionalmente aplicada no contexto de escalonamento de aeronaves. A adaptação dessa rede ao escalonamento de veículos 
é recente, mas já demonstra vantagens, em termos de menor dimensão, se comparada à rede baseada em conexões, amplamente utilizada. Por possuir rede de menor tamanho, a TSN pode auxiliar no desenvolvimento de modelos inovadores e menos complexos, com métodos de solução mais rápidos.

Os resultados do modelo VTSP-SCT possibilitaram economia no número de veículos, configurando-se como versátil no suporte à tomada de decisão no planejamento do transporte público. Isso porque, dadas as características das tabelas de horário geradas em cada configuração de intervalo de tempo $\Delta t$, pode-se definir qual adotar a fim de garantir um menor número de ônibus trafegando sem prejudicar a qualidade do atendimento prestado. Tratando-se especificamente dos resultados da aplicação do modelo às instâncias reais, observou-se redução de até 18 veículos na instância de maior porte ao intervalo $\Delta t=3$ minutos. Esse resultado é de grande valia para a operação do sistema de transporte público, tendo em vista que a sutil alteração na tabela de horários tende a economizar uma grande quantidade de veículos.

Oportunidades de pesquisas surgem a partir da realização deste estudo, podendo contribuir ainda mais com a otimização do planejamento do transporte público. A primeira delas reside na inclusão de múltiplas garagens (MD-VSP) ao problema proposto, visto que, na maioria das cidades de médio e grande porte, os ônibus são armazenados em mais de um local, facilitando o deslocamento dos veículos para o atendimento da população. Outra perspectiva recai sobre a integração dos problemas propostos com o problema de escalonamento da tripulação (crew scheduling), pois os custos incorridos da tripulação são ainda maiores do que aqueles oriundos do uso de veículos. Pesquisas que integram os problemas de escalonamento de veículos e tripulação têm sido desenvolvidas nos últimos anos, dada a importância dessas duas etapas para o planejamento operacional do transporte público. Entretanto, não se encontram publicados, até o momento, estudos que integrem esses dois problemas com a geração da tabela de horários e a frota heterogênea.

\section{REFERÊNCIAS}

ASSOCIAÇÃO NACIONAL DOS TRANSPORTES PÚBLICOS (ANTP). Sistema de Informações da Mobilidade Urbana. Relatório Geral 2011. São Paulo: ANTP, 2012. Disponível em: <http:// www.antp.org.br/_5dotSystem/download/ dcmDocument/2013/04/11/050FC84C-74EA4A33-A919-6D2E380FA2C1.pdf>. Acesso em: 13 dez. 2013.

BERTOSSI, A. A.; CARRARESI, P.; GALLO, G. On some matching problems arising in vehicle scheduling models. Networks, v. 17, p. 271281, 1987.

BUNTE, S.; KLIEWER, N. An overview on vehicle scheduling models in public transport. Public Transport, v. 1, n. 4, p. 299-317, 2009.

CEDER, A. Public-transport vehicle scheduling with multi vehicle type. Transportation Research Part C, v. 19, p. 485-497, 2011.

CEDER, A.; HASSOLD, S.; DANO, B. Approaching even-load and even-headway transit timetables using different bus sizes. Public Transport, v. 5, n. 3, p. 193-217, 2013.

DE LEONE, R; FESTA, P.; MARCHITTO, E. A Bus Driver Scheduling Problem: a new mathematical model and a GRASP approximate solution. Journal of Heuristics, v. 17, p. 441466, 2011.

DESAULNIERS, G.; LAVIGNE, J.; SOUMIS, F. Multi-Depot Vehicle Scheduling Problems with Time Windows and Waiting Costs. European Journal of Operational Research, v. 111, p. 479-494, 1998.

FRELING, R.; WAGELMANS, A. P. M.; PAIXÃO, J. M. P. An overview of models and techniques 
for integrating vehicle and crew scheduling. In: WILSON, N. H. M. (Ed.). Computer-aided Transit Scheduling, Lecture Notes in Economics and Mathematical Systems, Springer, v. 471, p. 441-460, 1999.

GUIHAIRE, V.; HAO, J-K. Transit network timetabling and vehicle assignment for regulating authorities. Computers \& Industrial Engineering, v. 59, n. 1, p. 16-23, 2010.

KLIEWER, N.; AMBERG, B.; AMBERG, B. Multiple depot vehicle and crew scheduling with time windows for scheduled trips. Public Transport, v. 3, n. 3, p. 213-244, 2012.

KLIEWER, N.; BUNTE, S.; SUHL, L. Time windows for scheduled trips in multiple depot vehicle scheduling. In: EURO Working Group on Transportation, Bari, 2006. Proceedings... Bari, 2006. p. 340-346.

KLIEWER, N.; MELLOULI, T.; SUHL, L. A new solution model for multi-depot multi-vehicletype vehicle scheduling in suburban public transport. In: Mini-EURO Conference and the EURO Woring Group on Transportation, 13., 2002, Bari. Proceedings... Bari, 2002. p. 604609.

KLIEWER, N.; MELLOULI, T.; SUHL, L. A TSN based exact optimization model for multidepot bus scheduling. European Journal of Operational Research, v. 175, n. 3, p. 1.6161.627, 2006.

MANHEIM, M. L. Fundamentals of transport system analysis. Boston: MIT Press, 1980. v. 1.

PRATA, B. A. Programação integrada de veículos e motoristas: uma visão geral. Revista Eletrônica Sistemas \& Gestão, v. 4, n. 3, p. 182204, 2009.

STEINZEN, I.; GINTNER, V.; SUHL, L. A timespace network approach for the integrated vehicle- and crew-scheduling problem with multiple depots. Transportation Science, Linthicum, v. 44, n. 3, p. 367-382, 2010. van den HEUVEL, A. P. R. V. D.; van den AKKER, J. M. V. D.; NIEKERK, M. E. V. K. Integrating timetabling and vehicle scheduling in public bus transportation. Technical Report. Department of Information and Computing Sciences, Utrecht University, Utrecht, The Netherlands, 2008. Disponível em: <http:// www.computerscience.nl/research/techreps/ repo/CS-2008/2008-003.pdf>. Acesso em: 10 nov. 2013.

VISENTINI, M. S. et al. A new implementation to the vehicle type scheduling problem with time windows for scheduled trips. In: Simpósio Brasileiro de Pesquisa Operacional, 45., Natal, 2013. Anais... Natal, 2013. 